\title{
Does splint therapy work for temporomandibular pain?
}

\author{
Is stabilisation splint therapy effective in reducing symptoms in people who \\ have pain dysfunction syndrome?
}

\begin{abstract}
Al-Ani MZ, Davies SJ, Gray RJM, Sloan P, Glenny AM. Stabilisation splint therapy for temporomandibular pain dysfunction syndrome (Cochrane Review). In the Cochrane Library. Chichester: John Wiley; 2004, Issue 1
\end{abstract}

Data sources The Cochrane Oral Health Group's Trials Register, the Cochrane Central Register of Controlled Trials (CENTRAL), Cochrane Library Issue 2 from 2003, Medline and Embase were all data sources. Relevant journals were also searched by hand and the reference lists of chosen studies were screened. Experts in the field were contacted and there were no language restrictions.

Study selection To be selected, the studies had to be randomised controlled trials (RCT) or quasi-RCT, in which splint therapy was compared concurrently with no treatment, other occlusal appliances or any other active intervention.

Data extraction and synthesis Data extraction was carried out independently and in duplicate. Validity assessment of the chosen trials was carried out at the same time as data extraction. Discrepancies were discussed and a third reviewer consulted. The author of the primary study was contacted where necessary. The studies were grouped according to treatment type and duration of follow-up.

Results Twenty potentially relevant RCT were identified. Eight were later excluded, leaving 12 trials for analysis. Stabilisation splint therapy (SS) was compared with: acupuncture, bite plates, biofeedback/stress management, visual feedback, relaxation, jaw exercises, non-occluding appliance and minimal/no treatment. There was no evidence of a statistically significant difference in the effectiveness of SS in reducing symptoms in patients with pain dysfunction syndrome (PDS) compared with other active treatments. There was weak evidence to suggest that the use of SS for the treatment of PDS may be beneficial for reducing pain severity, at rest and on palpation, compared with no treatment.

Conclusions There is insufficient evidence either for or against the use of SS for the treatment of temporomandibular PDS. This review suggests the need for further, rigorous RCT that consider the method of allocation and outcome assessment, have large sample size and sufficient duration of follow-up. A standardisation of the outcomes of the treatment of PDS should be established in the RCT.

Address for correspondence: Emma Tavender, Review Group Co-ordinator, Cochrane Oral Health Group, University Dental Hospital of Manchester, Higher Cambridge Street, Manchester M15 6FH, UK. E-mail: emma.tavender@man.ac.uk

\section{Commentary}

SS is commonly used by both general practitioner and specialists in their treatments for temporomandibular disorders (TMD). In this review the objective was to establish the effectiveness of SS in reducing symptoms in patients with PDS.

To evaluate the effectiveness of a treatment it is of utmost importance to have a diagnosis, towards which the treatment is directed. Schwartz ${ }^{1}$ described pain dysfunction as a syndrome in 1956, it being later defined in a study by Davies and Gray $^{2}$ through presence of at least two out of four criteria: pain on palpation of the temporomandibular joint, pain on palpation of associated mandibular muscles, limitation or deviation of mandibular movements and joint sounds and/or headache, both of which may or may not be a symptom. This syndrome excludes the most common diagnosis among TMD patients - myofacial pain - which has been defined by a couple of widely accepted diagnostic systems. ${ }^{3,4}$ The subdiagnoses have been shown to have a good reliability. ${ }^{3}$

It is surprising that this Cochrane review chose to define a syndrome where reliable and valid criteria are lacking. The highest level of evidence for therapy is systematic reviews and metaanalyses. In many areas such reviews are rare but Forsell et al. ${ }^{5}$ considered this topic in 1999, a fact not considered by the review. This is disappointing because the earlier review included a number of good trials not included in this more recent one.

Twenty trials were identified and the results were drawn from 12 trials. Most of the eight excluded were either not PDS or not specifically PDS. Those trials included were of different qualities and the outcome measures differed. In some of the trials the data were too limited for statistical evaluation. Most of the studies did not have blind outcome assessment. One of the two studies with blind outcome assessment included a 24-h treatment with the appliance therapies, which was not the case in the other treatment studies. Taking all the shortcomings into consideration, the review's conclusion is not surprising.

\section{Practice point}

- Until we have further evidence the practitioner can assist the patient by regarding SS as an interesting reversible treatment modality and part of symptomatic treatment.

Dr's Ziad Al-Ani and Robin Gray have commented for the authors of the original review:

- In 1955, Schwartz ${ }^{6}$ reported that he was able to delineate from the mass of patients with a conglomeration of TMJ symptoms a more definitive group of individuals whose problem was characterised by painful, limited mandibular movement, due in his opinion, to spasm in masticatory musculature. He applied the term "temporomandibular joint pain dysfunction syndrome" to this condition. The studies of Schwartz had a profound influence on the thinking of many individuals interested in TMD problems and produced the first major shift away from the narrow mechanical concept of an occlusal aetiology to the broader implication of the entire masticatory apparatus, as well as to the psychological 
characteristics of the patient. This definition was fully supported later by Laskin. ${ }^{7}$

- Our definition of PDS does not exclude myofacial pain as a main clinical finding because it clearly includes TMJ pain and pain of associated muscles as the most common symptoms of this condition.

- The review by Forssell et $a l^{5}$ considered all RCTs of occlusal treatments for temproromandibular disoreds. TMD is a general term which involves disorders of myogenous and arthrogenous as well as TMJ degenerative disease. Their review was not specifically on the use of stabilisation splint in the management of PDS. This was the reason for excluding many of their included studies.

- The difference in the methodology used by the available randomised controlled trials is the main outcome of our systematic review and that was the reason for stating in the conclusion "the need for further, rigorous RCT that consider the method of allocation and outcome assessment, have large sample size and sufficient duration of follow-up. A standardisation of the outcomes of the treatment of PDS should be established in future RCTs".

\section{Maria Nilner}

Faculty of Odontology, Malmö University, Malmö, Sweden
1. Schwartz LL. A temporomandibular joint pain-dysfunction syndrome. J Chronic Dis 1956; 3:284-293.

2. Davies SJ, Gray RJ. The pattern of splint usage in the management of two common temporomandibular disorders. Part II. The stabilisation splint in the treatment of pain dysfunction syndrome. Br Dent J 1997; 183:247-251.

3. Dworkin FD, LeResche L. Research diagnostic criteria for temporomandibular disorders: review, criteria, examinations and specifications, critique. J Craniomandib Disord Facial Oral Pain 1992; 4:301-355.

4. Okesson JP (Ed). Orofacial Pain. Guidelines for Assessment, Diagnosis and Management. Chicago: Quintessence Publishing; 1996.

5. Forsell H, Kalso E, Koskela P, Vehmanen R, Pukka P, Alanen P. Occlusal treatments in temporomandibular disorders: a qualitative systematic review of randomised controlled trials. Pain 1999; 83:549-560.

6. Schwartz L. Pain associated with the temporomandibular joint. J Am Dent Assoc 1955; 51:394.

7. Laskin D. Aetiology of the pain-dysfunction syndrome. J Am Dent Assoc 1969; 79:147.

Evidence-Based Dentistry (2004) 5, 65-66.

doi:10.1038/sj.ebd.6400266 